\title{
RADIOCARBON DATING OF ALKENONES FROM MARINE SEDIMENTS: II. ASSESSMENT OF CARBON PROCESS BLANKS
}

\author{
Gesine Mollenhauer ${ }^{1} \bullet$ Daniel Montluçon • Timothy I Eglinton \\ Department of Marine Chemistry and Geochemistry, Woods Hole Oceanographic Institution, Woods Hole, Massachusetts \\ 02543-1543, USA.
}

\begin{abstract}
We evaluate potential process blanks associated with radiocarbon measurement of microgram to milligram quantities of alkenones at the National Ocean Sciences Accelerator Mass Spectrometry (NOSAMS) facility. Two strategies to constrain the contribution of blanks to alkenone ${ }^{14} \mathrm{C}$ dates were followed: 1) dating of samples of known age and 2) multiple measurements of identical samples. We show that the potential contamination associated with the procedure does not lead to a systematic bias of the results of alkenone dating to either younger or older ages. Our results indicate that alkenones record $\Delta^{14} \mathrm{C}$ of ambient DIC with an accuracy of approximately $10 \%$. A conservative estimate of measurement precision is $17 \%$ for modern samples. Alkenone ${ }^{14} \mathrm{C}$ ages are expected to be reliable within $500 \mathrm{yr}$ for samples younger than $10,500{ }^{14} \mathrm{C} \mathrm{yr}$.
\end{abstract}

\section{INTRODUCTION}

Recent developments in organic geochemical preparative methods and in procedures for sample preparation and analysis using accelerator mass spectrometry (AMS) have made molecular-level radiocarbon dating possible (Eglinton et al. 1996, 1997; Pearson et al. 1998; von Reden et al. 1998). Applications of these techniques serve many different purposes in environmental, marine, microbiological, and paleoclimatological sciences (e.g. Pearson and Eglinton 2000; Pearson et al. 2000; Ohkouchi et al. 2002, 2003; Reddy et al. 2002; Teuten et al. 2005). ${ }^{14} \mathrm{C}$ signatures of individual organic compounds can be used to obtain information about sources, pathways, and residence times of these compounds as well as metabolic processes. Compound-specific ${ }^{14} \mathrm{C}$ dating may also be used for stratigraphic purposes in deep-sea sediments (Ohkouchi et al. 2003; Ingalls et al. 2004). If ${ }^{14} \mathrm{C}$ signatures of organic compounds are to be used to develop stratigraphies and as tracers of ${ }^{14} \mathrm{C}$ content of dissolved inorganic carbon in surface ocean waters (Pearson et al. 2000; Smittenberg et al. 2004), high-precision measurements are required.

Alkenones, a suite of long-chain unsaturated ketones, are synthesized by a well-identified group of marine prymnesiophyte algal species, notably the cosmopolitan coccolithophorid Emiliania huxleyi. Alkenones are virtually ubiquitous and exclusively of algal origin, which makes them useful biomarkers for the study of marine systems. These compounds have found particular value because the degree of unsaturation in alkenones varies as a function of growth temperature. This attribute, together with the preservation of this signal in oceanic sediments, has resulted in the widespread use of alkenones as a paleo-sea surface temperature proxy. In addition to these attributes, the source specificity of alkenones and other biomarkers of marine phytoplanktonic origin renders them potentially effective tracers of surface ocean dissolved inorganic carbon (DIC) ${ }^{14} \mathrm{C}$ content, and hence tools for developing sediment chronologies (Pearson et al. 2000; Ohkouchi et al. 2003). Comparison of the ${ }^{14} \mathrm{C}$ ages of molecular biomarkers and planktonic foraminiferal carbonate also provides an important means to assess temporal relationships between corresponding proxy records (Ohkouchi et al. 2002; Mollenhauer et al. 2003, 2005).

Before molecular ${ }^{14} \mathrm{C}$ dating can be routinely applied to address such problems, however, methods are required for effective purification of the analytes in question. In a companion paper, a procedure for isolation and purification of alkenones from marine sediments has been described (Ohkouchi et al., this issue). Moreover, because the amount of carbon available for such measurements is typi-

${ }^{1}$ Corresponding author. Email: gmollenhauer@whoi.edu. 
cally small, potential influences of the purification and analysis procedure on the resulting ${ }^{14} \mathrm{C}$ data must be carefully evaluated. For example, in some cases alkenones have yielded significantly older ${ }^{14} \mathrm{C}$ ages than planktic foraminifera from the same depth interval despite the common habitat of alkenone producers and planktic foraminifera in marine surface waters. The age offsets are interpreted to be the result of advection of significant amounts of pre-aged material (Ohkouchi et al. 2002; Mollenhauer et al. 2003, 2005). However, these observations of alkenones older than co-occurring foraminifera have raised concerns as to whether compound-specific ${ }^{14} \mathrm{C}$ ages may be biased due to the addition of ${ }^{14} \mathrm{C}$-depleted carbon during processing. Here, we refer to this carbon as the process blank.

Once purified, alkenones as well as other single compound or compound class samples targeted for ${ }^{14} \mathrm{C}$ analysis are combusted and processed in an analogous fashion to bulk organic matter samples (McNichol et al. 1994). However, most of these samples contain less than $300 \mu \mathrm{g}$ of carbon, and as such may require special protocols for AMS analysis of "small" samples. This includes simultaneous measurement of standards that closely bracket the size of the unknowns (Pearson et al. 1998). This approach has been adopted at the National Ocean Science Accelerator Mass Spectrometer (NOSAMS) facility in Woods Hole, USA.

In addition to potentially greater uncertainties associated with AMS analysis of small samples, incorporation of contamination during sample processing is a greater concern than for regular-sized samples. For example, contamination of single-compound and compound-class samples could stem from the elaborate purification techniques. Alkenone samples are purified using a sequence of wet chemical steps (cf. Ohkouchi et al., this issue). The samples are treated with large volumes of organic solvents, which typically are derived from petroleum products and thus do not contain any measurable ${ }^{14} \mathrm{C}$. Traces of these solvents or associated impurities remaining in the samples could potentially bias the data towards old ages. Secondly, it is possible that trace quantities of carboncontaining compounds that fall outside the "analytical window" of a gas chromatograph (GC) could be present. These would not be recognized when monitoring the sample purity using a GC. The combustion process represents a third potential source of contamination, which is a consideration for all other organic carbon samples. For example, the presence of dust particles in the pre-combusted quartz glass tubes could contribute $\mathrm{CO}_{2}$ during combustion. Such dust particles could have either modern ${ }^{14} \mathrm{C}$ signatures in the case of a natural source, or could contain synthetic fibers, which could be ${ }^{14} \mathrm{C}$-depleted, in variable relative amounts. Additional potential sources of blank carbon include sorption of $\mathrm{CO}_{2}$ to quartz tube walls; minor leaks in the vacuum line used for flame sealing the samples, which could introduce atmospheric $\mathrm{CO}_{2}$ and would thus be a source for a modern contamination; and addition of carbon during graphitization and during preparation of graphite targets.

In this study, we evaluate potential process blanks associated with ${ }^{14} \mathrm{C}$ measurement of microgram to milligram quantities of alkenones by AMS. We have followed 2 strategies to constrain the contribution of blanks to alkenone ${ }^{14} \mathrm{C}$ dates: 1) dating of samples of known age and 2) multiple measurements of identical samples. We show that there is no systematic contamination associated with the purification procedure that would bias the results of alkenone dating to either younger or older ages. Instead, alkenone samples of known age yield reliable results within measurement uncertainties, and reproducibility of measured results is good.

\section{MATERIALS AND METHODS}

The following types of samples were used to evaluate the potential sources and magnitudes of carbon blanks: 


\section{Samples of Known Age from Laboratory Cultures}

Two batch cultures of Isochrysis sp., a non-calcifying prymnesiophyte, were grown in the laboratory until the populations collapsed. Culture vessels, hoses, and filters were sterilized by autoclaving. Batch cultures were initiated by injecting a small $(\sim 5 \mathrm{~mL})$ inoculum into $15 \mathrm{~L}$ of ultrafiltered $(0.2-\mu \mathrm{m}$ filter pore size) seawater. Nutrients (marine water enrichment solution, $50 \times$, Guillard's [f 2], $300 \mathrm{~mL}$ ) were added in excess in order to stimulate rapid growth. One batch was grown in contact with the ambient atmosphere.

The second batch was grown in a ${ }^{14} \mathrm{C}$-depleted environment. For this experiment, seawater DIC was removed by adding an equivalent amount of protons (we assumed alkalinity of seawater $\sim 2 / \mathrm{mmol} / \mathrm{L}$ and added $2.717 \mathrm{~mL}$ of $12.1 \mathrm{M} \mathrm{HCl}$ into $15 \mathrm{~L}$ of seawater) and subsequently bubbling with nitrogen. Exchange with the atmosphere was minimized by sealing the culture vessel with a rubber stopper and restricting the gas flow to a small diameter glass tube, which was inserted through the rubber stopper. The glass tube was capped with a mildly hydrophobic glass-fiber filter (Whatman HEPA-Vent ${ }^{\circledR}, 0.3-\mu \mathrm{m}$ pore size), further limiting the exchange with the ambient atmosphere and preventing the introduction of bacteria. Alkalinity was restored by adding $\mathrm{NaOH}(1.315 \mathrm{~g})$ in equivalent molar quantity to neutralize the previously added acid, and bubbling with ${ }^{14} \mathrm{C}$-free $\mathrm{CO}_{2}$ and ${ }^{14} \mathrm{C}$-free air (mixture containing nitrogen, oxygen, and $\sim 300 \mathrm{ppm}{ }^{14} \mathrm{C}$-free $\mathrm{CO}_{2}$ ). After $\mathrm{pH}$ and alkalinity had been readjusted to near-normal seawater values $(\mathrm{pH} 7.53,2.27 \mathrm{mmol} / \mathrm{L})$, the culture was inoculated by adding a weighed aliquot of a cell culture of known ${ }^{14} \mathrm{C}$ content $(6.28 \mathrm{~mL}$, containing $1.79 \mathrm{mg}$ of Isochrysis sp. cells). Water samples were analyzed for ${ }^{14} \mathrm{C}$ content of DIC. DIC from the ${ }^{14} \mathrm{C}-$ depleted culture water was analyzed prior to inoculation and after the end of the experiment. After algal growth had reached a stationary phase or had stopped, the bottle content was filtered onto solvent-rinsed glass fiber filters (GF/F). Particulate matter on the filters was solvent extracted by ultrasonication (dichloromethane and methanol, 93:7, $1 \mathrm{~L}$ for $2 \mathrm{hr}, 1 \mathrm{~L}$ for $1 \mathrm{hr}, 1 \mathrm{~L}$ for $30 \mathrm{~min}$ ). Alkenones were purified and prepared for ${ }^{14} \mathrm{C}$ analysis according to the methods described by Ohkouchi et al. (this issue). This involved using a sequence of saponification, silica gel chromatography, and urea adduction purification steps. Subsamples containing variable amounts of alkenones were then combusted for ${ }^{14} \mathrm{C}$ target preparation.

\section{Sample of Known Age from a Field Experiment}

A stimulated Emiliania huxleyi bloom experiment was performed in June 2001 at the University of Bergen Biological Station, Norway (cf. Engel et al. 2005). Particulate matter accumulating at the bottom of a mesocosm bag was collected after the end of the experiment. This sample was solvent extracted by ultrasonication (DCM:Methanol 93:7, 3×15 min) and alkenones were purified at the University of Oldenburg, Germany. The purified sample was then ${ }^{14} \mathrm{C}$ dated along with DIC extracted from water samples collected on days 1, 9, and 22 of the experiment. These analyses were carried out at the Leibniz-Laboratory in Kiel, Germany.

\section{Sample of Known Age from Sediments}

Alkenones were extracted from sediments at the 1013-1031-cm depth interval of marine sediment core IOW226920-3 off Namibia (Mollenhauer et al. 2003). Extrapolation of the oxygen isotope stratigraphy for the upper $330 \mathrm{~cm}$ of this core places this core depth at Marine Isotope Stage 5e or earlier (i.e. >120,000 BP; Mollenhauer et al. 2003). Extraction and purification procedures were as described by Ohkouchi et al. (this issue). Two of these alkenone samples were processed in Oldenburg and measured in Kiel. One sample was processed and measured in Woods Hole (NOSAMS). 


\section{Replicate Measurements of Sediment Samples of Variable Ages}

Whenever sufficient amounts of alkenones could be purified from sediment samples studied in a separate project (Mollenhauer and Eglinton, unpublished data), the purified fractions were split in 2 or 3 subsamples and dated individually. Two of the multiply dated samples were split after total lipid extraction and have subsequently been purified individually. Some results from these analyses have previously been reported (Mollenhauer et al. 2003, 2005). The multiple analyses performed on splits of purified samples do not provide information about possible blanks associated with the purification procedure. However, possible combustion, graphitization, and measurement blanks could be traced by this method since the relative contribution of a constant carbon blank should vary with sample size. Two of the samples split after purification were prepared on 2 different vacuum lines in order to detect possible blanks deriving from minor leaks in either vacuum system.

\section{RESULTS AND DISCUSSION}

Results of ${ }^{14} \mathrm{C}$ measurements of the samples with known age are given in Table 1, and results of replicate analyses are presented in Table 2. NOSAMS results are reported without combustion blank correction. The only correction applied is based on simultaneous measurement of small standards (matching the analytes' sample sizes) that are prepared from a large homogenous gas standard (Pearson et al. 1998). In this way, AMS effects resulting in depleted values for small graphite targets, when normalized to regular-sized standard material, are avoided (cf. McNichol et al. 2001).

\section{Alkenones from Culture Experiments}

In order to detect a potential contribution of fossil blank carbon, 10 subsamples were analyzed of the purified alkenones isolated from the Isochrysis sp. culture grown under ambient laboratory air conditions ranging in size from over $2 \mathrm{mg}$ to $47 \mu \mathrm{g}$ carbon. These samples should contain modern atmospheric levels of ${ }^{14} \mathrm{C}$. The results of the 10 analyses agree both with the DIC extracted from the water and with each other (Figure 1, Table 1). Alkenone $\Delta{ }^{14} \mathrm{C}$ ranges from 29 to $87 \%$, while $\Delta{ }^{14} \mathrm{C}$ of the DIC is $62 \%$. The average of all alkenone $\Delta^{14} \mathrm{C}$ is within $2 \%$ of the DIC value, which is well within the measurement uncertainty. The maximum deviation of alkenone $\Delta^{14} \mathrm{C}$ from the DIC value amounts to $33 \%$ and occurs in a sample containing $>500 \mu \mathrm{g}$ carbon, which is thus considered a "regular-sized" sample. The standard deviation of the entire data set $(n=10)$ is 17\%o. This estimate of standard deviation is similar to that obtained for small organic standards run routinely at NOSAMS (McNichol, unpublished data).

In this data set, no evidence for the presence of a ${ }^{14} \mathrm{C}$-depleted blank contribution is observed, which would potentially shift "small" sample results to lower values. Instead, 2 of the 5 small samples (i.e. $<300 \mu \mathrm{g} \mathrm{C}$ ) had higher ${ }^{14} \mathrm{C}$ concentrations than the DIC of the culture medium, indicating the possibility of a small contribution of a ${ }^{14} \mathrm{C}$-rich blank or the occurrence of an effect related to the measurement. This tendency towards higher measured ${ }^{14} \mathrm{C}$ concentrations in small samples is not correlated to sample size and is due to the small number of samples analyzed not statistically significant. A similar trend towards ${ }^{14} \mathrm{C}$ enrichment is also observed when "small" targets are prepared of international standard reference material for AMS analyses (McNichol, unpublished data). The origins of the shift are currently under investigation (see discussion below).

We explored the possibility of a modern process blank by analyzing 7 subsamples of alkenones purified from the batch culture prepared with ${ }^{14} \mathrm{C}$-free artificial air $\left(\mathrm{CO}_{2}: \Delta{ }^{14} \mathrm{C}=-997.8 \pm 0.8 \%\right.$ o $)$. Sample sizes ranged from 709 to $39 \mu \mathrm{g}$ (Figure 2, Table 1). Three measurements of DIC $\Delta^{14} \mathrm{C}$ were performed as well. One water sample was taken before and 2 were taken after the culture 


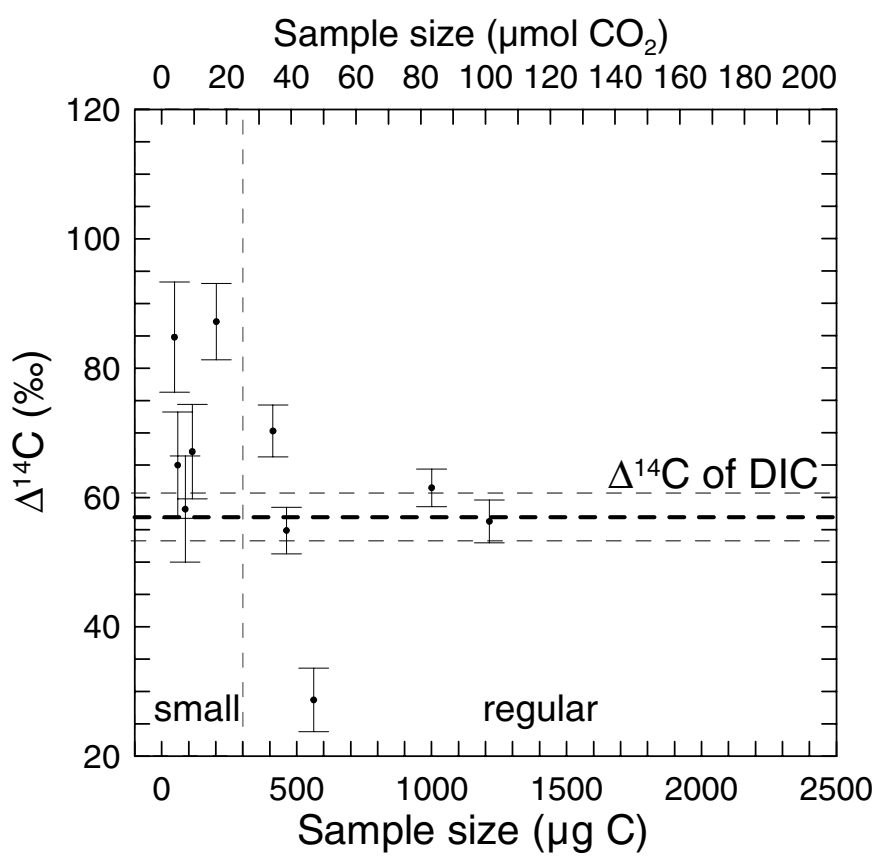

Figure $1 \Delta^{14} \mathrm{C}$ of alkenones obtained from a culture of Isochrysis sp. grown in contact with laboratory air plotted versus sample size. Error bars are 1- $\sigma$ values. $\Delta{ }^{14} \mathrm{C}$ of DIC in the culture water is given as the thick dashed line; $1-\sigma$ confidence limits are indicated by the thin dashed lines. The vertical dashed line separates samples measured as small samples and regular-sized samples.

experiment. One of the post-experiment samples is from the unfiltered water, while the second was taken after suspended algal cells had been recovered by filtering. The measurements yielded $\Delta^{14} \mathrm{C}$ of $-877 \pm 1 \%$ o before the experiment, $-843 \pm 1 \%$ o in the unfiltered water sample, and $-828 \pm 1 \%$ o for the filtered water. These results indicate that (a) some exchange between the culture water and the ambient atmosphere occurred prior to inoculation and during the course of the experiment, contributing approximately $10 \%$ of the total dissolved $\mathrm{CO}_{2}$ species; and (b) further exchange with the atmosphere during filtering increased the ${ }^{14} \mathrm{C}$ content of the third sample. The measured ${ }^{14} \mathrm{C}$ concentration in the alkenones obtained from this second culture experiment can thus not be used to test whether alkenones faithfully record $\mathrm{DI}^{14} \mathrm{C}$ of the seawater in which the algae grew. However, the analysis of a suite of different-sized subsamples of a homogeneous amount of purified alkenones would allow detection of potential systematic addition of modern carbon during sample combustion as a function of sample size.

Two of the alkenone samples from the ${ }^{14} \mathrm{C}$-depleted culture experiment were "regular-sized" samples (>300 $\mu \mathrm{g} \mathrm{C})$ and analyzed using standard AMS procedures (McNichol et al. 1994). They yielded identical results within $1-\sigma$ error margins. The 5 subsamples of the culture-derived purified alkenones analyzed as "small" samples also yielded identical results within 1- $\sigma$ errors. This "small" subset was enriched in ${ }^{14} \mathrm{C}$ by about $9 \%$ when compared to the "regular-sized" subset. The "small" and "regular-sized" sample subsets, however, agreed within 2- $\sigma$ errors. Standard deviation of the entire data set $(n=7)$ was 5\%. The offset between small and regular-sized sample subsets may stem from different data analysis procedures for "small" samples. 
Table $1 \Delta^{14} \mathrm{C}$ of samples of known age. Average value for modern Isochrysis sp. alkenones, regular-sized samples, is given excluding sample Isochrysis sp. alkenones 3 (OS-40250). The value in parentheses includes all results. Alkenone $\Delta^{14} \mathrm{C}$ of KIA19294, KIA19292, and KIA19293 (printed in italic font) are reported as corrected values after background correction of $0.0246 \mathrm{fm} \mathrm{C}, 0.0268 \mathrm{fm} \mathrm{C}$, and $0.9284 \mathrm{fm} \mathrm{C}$, respectively.

\begin{tabular}{|c|c|c|c|c|}
\hline Sample & Lab ID & $\begin{array}{l}\text { Sample size } \\
(\mu \mathrm{g} \mathrm{C})\end{array}$ & $\Delta^{14} \mathrm{C}(\% \circ)$ & $\begin{array}{l}\Delta^{14} \mathrm{C} \\
\text { error }(\% o)\end{array}$ \\
\hline \multicolumn{5}{|l|}{ Culture experiment I, ambient air } \\
\hline DIC & OS-38352 & & 61.5 & 2.9 \\
\hline Isochrysis sp. alkenones 0 & OS-37730 & 462 & 54.9 & 3.6 \\
\hline Isochrysis sp. alkenones 1 & OS-40248 & 2203 & 57.0 & 3.7 \\
\hline Isochrysis sp. alkenones 2 & OS-40249 & 1214 & 56.3 & 3.3 \\
\hline Isochrysis sp. alkenones 3 & OS-40250 & 563 & $(28.7)$ & 4.9 \\
\hline Isochrysis sp. alkenones 4 & OS-40269 & 412 & 70.3 & 4.0 \\
\hline Isochrysis sp. alkenones 5 & OS-40286 & 202 & 87.2 & 5.9 \\
\hline Isochrysis sp. alkenones D & OS-40299 & 113 & 67.1 & 7.3 \\
\hline Isochrysis sp. alkenones $\mathrm{C}$ & OS-40304 & 87 & 58.2 & 8.2 \\
\hline Isochrysis sp. alkenones B & OS-40308 & 59 & 65.0 & 8.2 \\
\hline \multirow[t]{2}{*}{ Isochrysis sp. alkenones A } & OS-40304 & 47 & 84.8 & 8.5 \\
\hline & & & & std. dev. \\
\hline average, regular-sized samples & & & $59.6(53.4)$ & $7.2(15.2)$ \\
\hline average, small samples & & & 72.5 & 12.8 \\
\hline average, all samples & & & 63.0 & 16.6 \\
\hline \multicolumn{5}{|l|}{ Culture experiment II, “dead air" } \\
\hline DIC pre-experiment & OS-44691 & & -876.5 & 0.8 \\
\hline DIC post-experiment, unfiltered water & OS-44582 & & -843.1 & 1.1 \\
\hline DIC post-experiment, filtered water & OS-44583 & & -827.7 & 1.3 \\
\hline Isochrysis sp. alkenones A & OS-45090 & 709 & -861.5 & 1.2 \\
\hline Isochrysis sp. alkenones $\mathrm{B}$ & OS-45067 & 359 & -859.6 & 1.6 \\
\hline Isochrysis sp. alkenones $\mathrm{C}$ & OS-45471 & 219 & -849.4 & 4.9 \\
\hline Isochrysis sp. alkenones D & OS-45481 & 146 & -855.5 & 3.4 \\
\hline Isochrysis sp. alkenones $\mathrm{E}$ & OS-45484 & 112 & -852.3 & 7.2 \\
\hline Isochrysis sp. alkenones $\mathrm{F}$ & OS-45490 & 57 & -851.5 & 7.4 \\
\hline Isochrysis sp. alkenones $\mathrm{G}$ & OS-45493 & 39 & -848.4 & 7.4 \\
\hline Culture inoculum (isochrysis cells, bulk OC) & OS-45469 & 229 & 97.4 & 1.27 \\
\hline $\begin{array}{l}\text { Culture inoculum (isochrysis cells, } \\
\text { bulk OC, acidified) }\end{array}$ & OS-45472 & 205 & 107.9 & 1.03 \\
\hline $\begin{array}{l}\text { average, regular-sized samples } \\
\text { average, small samples } \\
\text { average, all samples }\end{array}$ & & & $\begin{array}{l}-860.6 \\
-851.4 \\
-854.0\end{array}$ & $\begin{array}{l}\text { std. dev. } \\
1.3 \\
2.8 \\
5.0\end{array}$ \\
\hline \multicolumn{5}{|l|}{ Field experiment, mesocosm in Fjord } \\
\hline DIC day 1 & KIA19297 & & 114.4 & 2.5 \\
\hline DIC day 9 & KIA19298 & & 133.1 & 3.1 \\
\hline DIC day 22 & KIA19299 & & 156.6 & 2.8 \\
\hline alkenones & KIA19294 & 180 & 105.2 & 8.9 \\
\hline \multicolumn{5}{|l|}{ IOW226920-3, 1013-1031 cm, pre-Eemian } \\
\hline alkenones Oldenburg/Kiel I & KIA19292 & 170 & -997.5 & 9.0 \\
\hline alkenones Oldenburg/Kiel II & KIA19293 & 160 & -998.0 & 9.5 \\
\hline alkenones Woods Hole & OS-37731 & 1164 & -995.7 & 0.2 \\
\hline
\end{tabular}




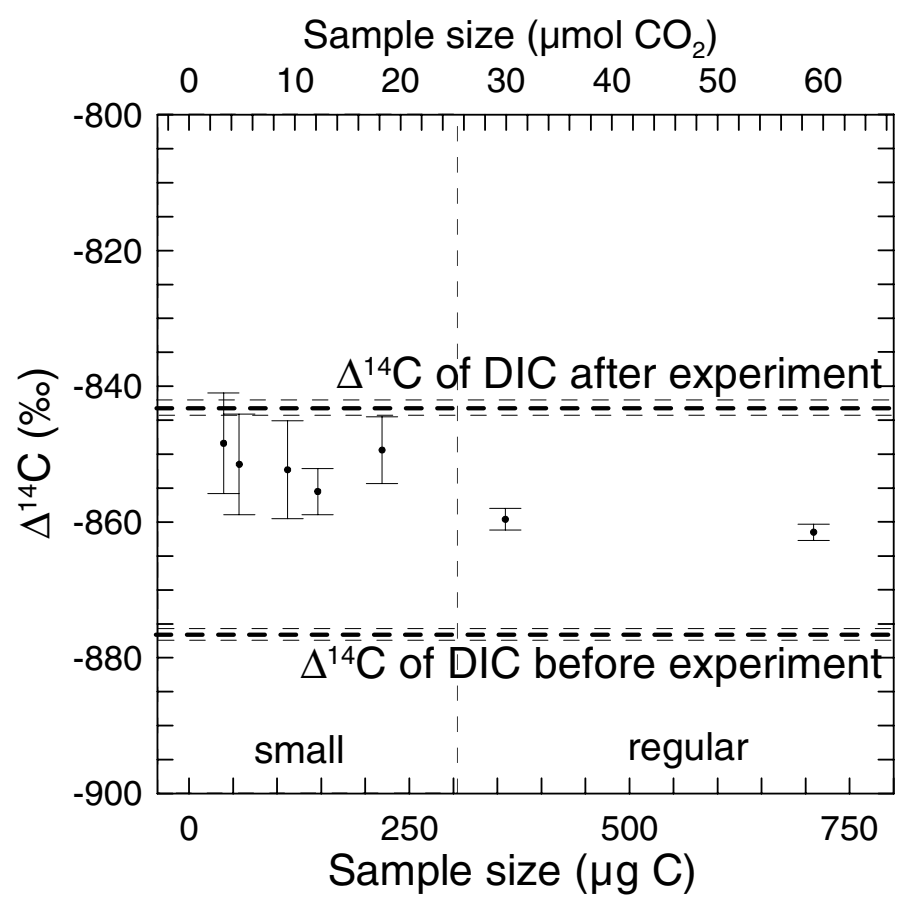

Figure $2 \Delta^{14} \mathrm{C}$ of alkenones obtained from a culture of Isochrysis sp. grown in a ${ }^{14} \mathrm{C}$-depleted medium (see text) plotted versus sample size. Error bars are 1- $\sigma$ values. $\Delta^{14} \mathrm{C}$ of DIC in the culture water before and after the experiment are given as the thick dashed lines; $1-\sigma$ confidence limits are indicated by the thin dashed lines. The vertical dashed line separates samples measured as small samples and regular-sized samples.

The observation of an offset towards higher $\Delta^{14} \mathrm{C}$ values for small samples of material from both cultures points to an AMS-related cause. The "near dead" sample set would be expected to be more sensitive to addition of modern blank carbon than the modern samples, which would result in a larger offset between small and regular-sized samples. Instead, the small sample subset of the modern culture material is more significantly enriched compared to the regular-sized sample subset of this batch. If the ${ }^{14} \mathrm{C}$ enrichment of the small samples derived from systematic addition of a modern process blank, the smallest samples would be expected to be affected the most. This is not observed, either. We therefore believe that the reasons for the enrichment of small samples lie in the AMS analysis, and that the problem appears to be more severe at higher ${ }^{14} \mathrm{C}$ levels. This potential AMS effect is not identical with the AMS effect causing lower ${ }^{14} \mathrm{C}$ values for small targets when normalizing to regular-sized standards as described previously (Pearson et al. 1998; McNichol et al. 2001). Our results are corrected for this effect by normalizing to size-matched standards. The processes leading to the offsets are currently under investigation at NOSAMS (McNichol, personal communication, 2005). The magnitude of the enrichment is small compared with age offsets between different sediment constituents, which often amount to 50 to $200 \%$ and larger, with small alkenone samples commonly older than regular-sized carbonate samples (cf. Ohkouchi et al. 2002; Mollenhauer et al. 2003, 2005). Therefore, the bias does not affect the applicability of the method for the study of, for example, sedimentation processes.

All alkenone subsamples from the ${ }^{14} \mathrm{C}$-depleted culture experiment had $\Delta{ }^{14} \mathrm{C}$ within the range of values obtained for $\mathrm{DI}^{14} \mathrm{C}$, further indicating that alkenones faithfully record ambient $\mathrm{DI}^{14} \mathrm{C}$ values. 
Cell numbers per volume were counted in the inoculum and in the culture upon termination of the experiment. The inoculum contained 3.89-4.00 $\times 10^{6}$ cells per $\mathrm{mL}$, while the 2 aliquots of the culture contained $1.44-1.55 \times 10^{6}$ cells per $\mathrm{mL}$ and $1.17-1.33 \times 10^{6}$ cells per $\mathrm{mL}$, respectively (each counted in duplicate). Based on these numbers, we calculate that approximately $25 \times 10^{6}$ cells were added to the $15 \mathrm{~L}$ of seawater $(6.28 \mathrm{~mL}$ of inoculum). By extrapolation of the cell concentrations counted in the aliquots taken from the culture upon termination, we estimate that the culture contained 1.5-2.0 $\times 10^{10}$ cells. In an independent approach to estimate the ratio of modern carbon stemming from the cells in the inoculum and carbon produced during the culturing experiment, we weighed both the biomass per volume in the inoculum $(0.28 \mathrm{mg} / \mathrm{mL})$ and the total biomass recovered from the culture $(874.7 \mathrm{mg})$. Since $6.28 \mathrm{~mL}$ of the inoculum was added, we estimate that $1.79 \mathrm{mg}$ of the total biomass recovered from the culture stems from the inoculum. Cell counts, as well as mass balance calculations based on cell weights, thus show that 0.12 and $0.2 \%$ of the total cells and cell weight, respectively, were inherited from the inoculum. $\Delta^{14} \mathrm{C}$ of the total organic matter derived from the inoculum was 97 to $108 \%$; the latter value was obtained from acidified biomass (Table 1). Assuming equal alkenone concentrations per unit total biomass or per cell in the algal detritus filtered from the culture as in the inoculum, we estimate that alkenones produced during the culture experiment should contain $\sim 2 \%$ o less ${ }^{14} \mathrm{C}$ than the value measured for samples containing a mixture of alkenones from the culture and from the inoculum. Given the uncertainty of the $\mathrm{DI}^{14} \mathrm{C}$ during the growth of the algae, the contribution of modern carbon deriving from the inoculum therefore is considered insignificant.

Data from the 2 sets of alkenone samples isolated from the culture experiments imply that the contribution of blank carbon associated with the alkenone purification procedure is insignificantly different from blank contribution to standard material and can therefore be corrected for. No evidence for a systematic additional contribution of either modern or fossil carbon blanks could be found.

\section{Field Experiment, Norwegian Fjord}

Alkenones extracted from the sedimented cells recovered from a mesocosm bag after the stimulation of an Emiliania huxleyi bloom were purified and ${ }^{14} \mathrm{C}$ dated at the Leibniz-Laboratory in Kiel, Germany. Water samples taken on days 1, 9, and 22 of the bloom experiment conducted in June 2001 were analyzed for DI ${ }^{14} \mathrm{C}$. All DIC samples have $\Delta^{14} \mathrm{C}$ levels in excess of the atmospheric value of the year of the experiment, which may point to a possible ${ }^{14} \mathrm{C}$ contamination of the experimental environment, possibly as a result of prior biological tracer studies. The research facility is open for biological experiments carried out by various research groups from all over Europe. The use of ${ }^{14} \mathrm{C}$ as a tracer thus seems a likely scenario responsible for the high ${ }^{14} \mathrm{C}$ concentrations of the samples. The alkenones seem to be affected to a much lesser extent, if at all, by such a possible contamination. This implies that the contamination occurred during handling at the biological field station in Norway or during storage of the water samples at the Alfred-Wegener-Institute in Bremerhaven, Germany. The origin of this potential contamination, however, cannot be further explored within this study.

The alkenone sample has the lowest $\Delta^{14} \mathrm{C}$ of all 4 samples analyzed (Table 1), while DIC of the last day of the experiment had the highest $\Delta^{14} \mathrm{C}$. Most alkenones are produced during the stationary phase of a coccolithophorid bloom (Prahl et al. 2003; Benthien A, Zondervan I, Riebesell U, Engel A, Hefter J, Delille B, Harlay J, Jacquet S, unpublished results), which implies that alkenones should be most similar in $\Delta^{14} \mathrm{C}$ to this last DIC sample. The difference between the measured $\Delta^{14} \mathrm{C}$ in these 2 samples amounts to $47 \%$ when using the uncorrected value for the alkenone sample. A large background correction is used at the Leibniz-Laboratory $(0.02464 \mathrm{fm} \mathrm{C}$ for the alkenone sample from the 
field experiment), which may imply that the difference between the $\Delta^{14} \mathrm{C}$ of day $22 \mathrm{DIC}$ and the alkenones may even be larger (52\%). Although this could indicate that there is a small bias towards lower $\Delta^{14} \mathrm{C}$ in alkenones with respect to the ambient seawater, no such bias could be detected in any of the other experiments reported here. Considering the uncertainty over the source for the excess ${ }^{14} \mathrm{C}$ found in the samples, as well as the uncertainty over when contamination occurred (e.g. during sample handling and storage), we consider that the agreement between all values obtained in this experiment is satisfactory. Moreover, the offset observed between alkenones and DIC of the last day of the bloom experiment is significantly lower than offsets in ${ }^{14} \mathrm{C}$ content between co-occurring foraminifera and alkenones observed in deep-sea sediments. Therefore, such a bias, if it occurs at all, would be too small to account for the reported age offsets in sediment samples, which for modern samples are often larger than 200\% (Ohkouchi et al. 2002; Mollenhauer et al. 2003).

\section{Alkenones Extracted from Sediments of Known Age}

Three measurements were obtained on alkenones extracted from homogenized sediment taken from the pre-Eemian (>120,000 yr) section of sediment core IOW226920-3. The 2 samples measured on the Kiel AMS were splits of a sample purified in Oldenburg. Blank corrections on the same order of magnitude as the measured signal were applied to the 2 samples $(0.0268$ and $0.0285 \mathrm{fm} \mathrm{C})$. Therefore, these 2 samples cannot be used to investigate a possible contribution of modern carbon. The third sample was obtained by a separate purification process and measured at the NOSAMS facility, and the result is reported as an uncorrected value. Comparison of data obtained using these 2 ways of sample analysis-i.e. background subtraction and simultaneous measurement of size-matched standard materials, respectively_-shows that the latter method is more appropriate for small sample analysis (cf. Pearson et al. 1998; McNichol et al. 2001).

All 3 measurements yielded very low $\Delta^{14} \mathrm{C}$ values (Table 1). The values are close to what would be considered a combined process and machine blank for "regular-sized" samples. A mass balance calculation suggests addition of 3-5 $\mu \mathrm{g}$ modern carbon to the largest sample (OS-37731), assuming the true value to be ${ }^{14} \mathrm{C}$-dead, which is similar to findings by Brown and Southon (1997) for the Lawrence Livermore National Laboratory facility. This reinforces the notion that modern blank carbon contribution associated with the elaborate alkenone purification procedure is small. Combined process and combustion blanks of compound-specific samples are similar to the standard sample combustion blank and can therefore be corrected for.

\section{Replicate Measurements of Samples}

In addition to the samples derived from the Isochrysis sp. cultures, a total of 8 purified alkenone samples from natural sediments yielded sufficient amounts of carbon to allow multiple measurements (Table 2, Figure 3). Their respective $\Delta^{14} \mathrm{C}$ values range from -890 to $-119 \%$ o. This large range of ages would allow the detection of a systematic combustion blank of any composition. Figure 3 also shows the multiple measurements of the 2 culture experiments (Figures 1 and 2).

Good agreement between sample replicates was achieved. Furthermore, there are no observable trends with sample size. Most replicates agree within the reported 1- $\sigma$ error margins. For those samples that have been dated in triplicate, there is 1 sample each that was dated slightly outside the error margins of the other two. However, in all cases but one, agreement is within 17\%o, the estimate of standard deviation from analysis of the alkenones derived from the modern Isochrysis sp. batch culture. 
Table $2 \Delta^{14} \mathrm{C}$ of replicate samples.

\begin{tabular}{lllll}
\hline & & $\begin{array}{l}\text { Sample size } \\
(\mu \mathrm{g} \mathrm{C})\end{array}$ & $\begin{array}{l}\Delta^{14} \mathrm{C} \\
(\%)\end{array}$ & $\begin{array}{l}\Delta^{14} \mathrm{C} \text { error } \\
(\% \circ)\end{array}$ \\
\hline Sample & Lab ID & & & \\
A (purified individually) & & & -875.0 & 4.4 \\
B1 (separated after combustion) & OS-31147 & 249 & -886.7 & 2.7 \\
B2 (separated after combustion) & OS-31154 & 156 & -879.7 & 3.4 \\
IOW226920, 230-232 (Mollenhauer et al. 2003) & OS-31156 & 156 & & \\
A & & & -931.6 & 2.5 \\
B (di-unsaturated alkenones only) & OS-31149 & 204 & -929.0 & 3.9 \\
GeoB3313-1, 153-158 (Mollenhauer et al. 2005) & OS-31164 & 97 & & \\
A (Fye) & OS-43130 & 261 & -288.9 & 21.8 \\
B (NOSAMS) & OS-43132 & 254 & -285.0 & 21.2 \\
GeoB3313-1, 633-638 (Mollenhauer et al. 2005) & & & & \\
A (Fye) & OS-43135 & 216 & -553.5 & 18.0 \\
B (NOSAMS) & OS- 43136 & 209 & -553.0 & 17.4 \\
NH01-12, MC40, 19-20 & & & & \\
A & OS-41847 & 144 & -176.7 & 6.4 \\
B & OS-41168 & 322 & -184.5 & 11.5 \\
NH01-12, MC50, 6-7 & & & & \\
A & OS-41851 & 121 & -137.6 & 5.8 \\
B & OS-41836 & 249 & -118.5 & 6.5 \\
C & OS-41169 & 346 & -137.8 & 10.0 \\
NH01-12, MC54, 5-6 & & & & \\
A & OS-41848 & 134 & -145.8 & 6.8 \\
B & OS-41835 & 266 & -132.8 & 7.1 \\
C & OS-41171 & 362 & -156.2 & 12.0 \\
\hline
\end{tabular}

Agreement between splits is also very good for those samples from core IOW226920 that were separated after total lipid extraction and purified individually. Moreover, due to problems during the purification procedure, one of the splits of sample IOW226920, 230-232 cm, contained di-unsaturated alkenones only, while in the second split both di- and tri-unsaturated species were present. Nevertheless, ${ }^{14} \mathrm{C}$ contents of both splits for this sample agree within the error margins.

\section{SUMMARY AND CONCLUSIONS}

This study evaluated precision and accuracy of ${ }^{14} \mathrm{C}$ dating of alkenones carried out at NOSAMS. Our results indicate that alkenones record ambient $\mathrm{DI}^{14} \mathrm{C}$ with an accuracy of approximately $10 \%$ when using the analytical procedures described above. No significant amounts of either modern or ${ }^{14} \mathrm{C}$-depleted blank carbon are systematically introduced during the purification procedures. This is true for compounds purified both from particulate material filtered from water samples and from sediment samples. Observed precision is similar to that determined in a separate study of small samples prepared from standard reference material. We consider a conservative estimate of measurement precision of $17 \%$ o for modern samples. Based on this estimate, alkenone ${ }^{14} \mathrm{C}$ ages obtained from this facility should be reliable within $500 \mathrm{yr}$ for samples younger than $10,500{ }^{14} \mathrm{C} \mathrm{yr}$. The superior precision observed for the alkenones obtained from the ${ }^{14} \mathrm{C}$-depleted culture experiment implies that alkenones from samples up to $20,000{ }^{14} \mathrm{C}$ yr old should be precise. Finally, replicate analyses of samples of variable sizes showed that there is no trend towards younger or older ages with sample size. 


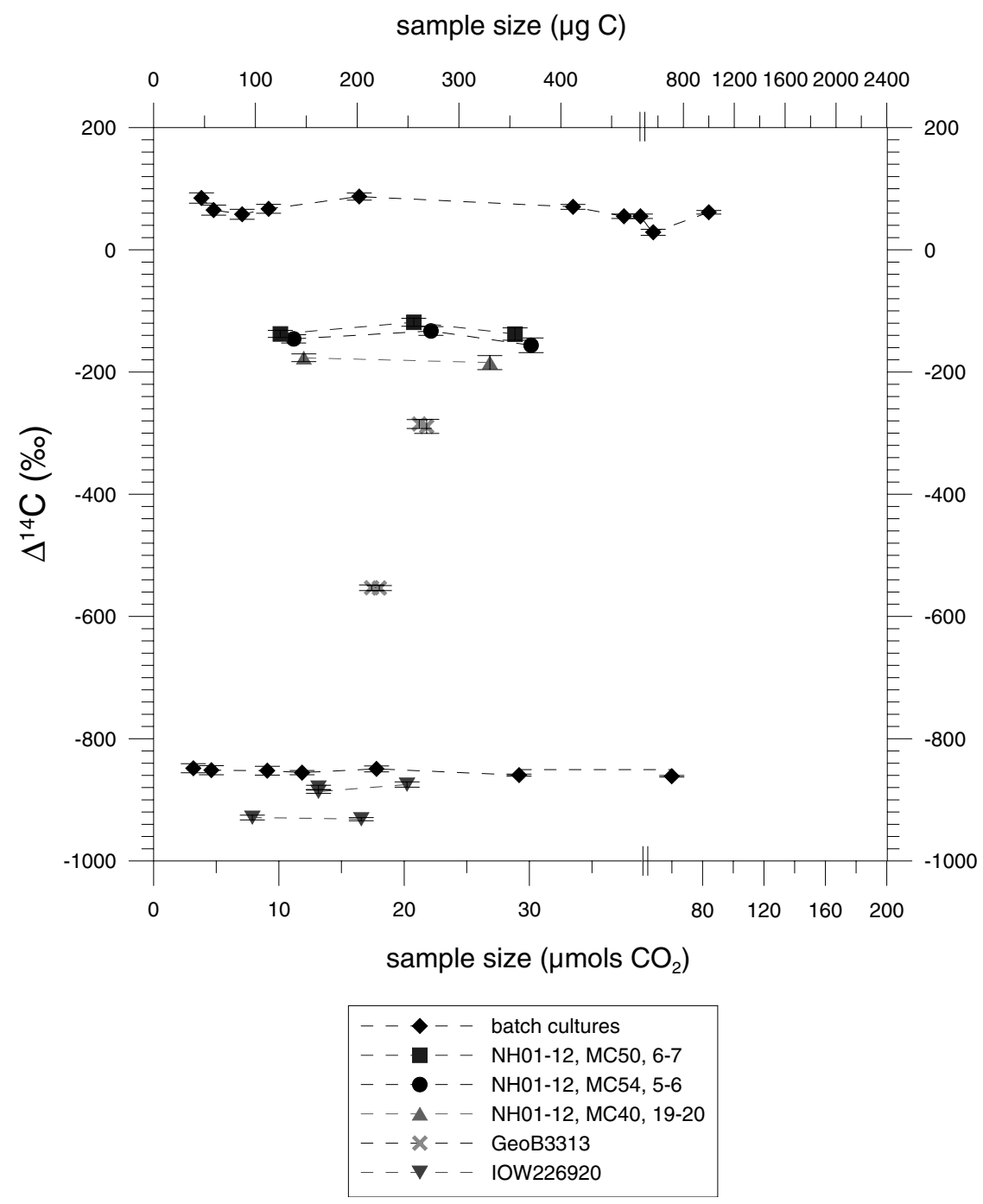

Figure $3 \Delta^{14} \mathrm{C}$ of sediment-derived alkenone samples measured in replicate plotted versus their respective size. For comparison, results from the culture experiments are given as well (diamonds). Replicates of identical samples are connected with dashed lines.

We conclude, therefore, that the methods developed for purification and molecular level alkenone ${ }^{14} \mathrm{C}$ dating are both feasible and reliable when combined with the NOSAMS small-sample AMS protocols involving simultaneous analysis of size-matched standard material. Where offsets to independently dated sediment fractions are observed, sedimentary processes must therefore be considered the most likely cause.

\section{ACKNOWLEDGMENTS}

We thank Li Xu and Marion Luttmann for assistance with sample preparation, Ann McNichol for discussion and data, Kathy Elder for data base support, the NOSAMS staff, and the Leibniz Laboratory staff. Albert Benthien and Ulf Riebesell provided sample material from the mesocosm exper- 
iment. Dan McCorkle and Becky Belastock assisted with carbonate system and DIC measurements. Dave Kulis provided the Isochrysis sp. inoculum and assisted with cell counts. Comments by Greg Hodgins and two anonymous reviewers helped improve the manuscript. This research was funded by NSF grant \# OCE-0327405 and DFG project \# SCHN621 and a WHOI-NOSAMS postdoctoral scholarship to GM.

\section{REFERENCES}

Brown TA, Southon J. 1997. Corrections for contamination background in AMS ${ }^{14} \mathrm{C}$ measurements. Nuclear Instruments and Methods in Physics Research B 123: 208-13.

Eglinton TI, Aluwihare LI, Bauer JE, Druffel ERM, McNichol AP. 1996. Gas chromatographic isolation of individual compounds from complex matrices for radiocarbon dating. Analytical Chemistry 68:904-12.

Eglinton TI, Benitez-Nelson BC, Pearson A, McNichol AP, Bauer JE, Druffel ERM. 1997. Variability in radiocarbon ages of individual organic compounds from marine sediments. Science 277:796-9.

Engel A, Zondervan I, Aerts K, Beaufort L, Benthien A, Chou L, Delille B, Gattuso J, Harlay J, Heemann C, Hoffmann L, Jacquet S, Nejstgaard J, Pizay M, Rochelle-Newall E, Schneider U, Terbrueggen A, Riebesell U. 2005. Testing the direct effect of $\mathrm{CO}_{2}$ concentration on a bloom of the coccolithophorid Emiliania huxleyi in mesocosm experiments. Limnology and Oceanography 50:493-507.

Ingalls A, Anderson RF, Pearson A. 2004. Radiocarbon dating of diatom-bound organic compounds. Marine Chemistry 92:91-105.

McNichol AP, Osborne EA, Gagnon AR, Fry B, Jones GA. 1994. TIC, TOC, DIC, DOC, PIC, POC - unique aspects in the preparation of oceanographic samples for ${ }^{14} \mathrm{C}$-AMS. Nuclear Instruments and Methods in Physics Research B 92:162-5.

McNichol AP, Jull AJT, Burr GS. 2001. Converting AMS data to radiocarbon values: considerations and conventions. Radiocarbon 43(2A):313-20.

Mollenhauer G, Eglinton TI, Ohkouchi N, Schneider RR, Müller PJ, Grootes PM, Rullkötter J. 2003. Asynchronous alkenone and foraminifera records from the Benguela Upwelling System. Geochimica et Cosmochimica Acta 67:2157-71.

Mollenhauer G, Kienast M, Lamy F, Meggers H, Schneider RR, Hayes JM, Eglinton TI. 2005. An evaluation of ${ }^{14} \mathrm{C}$ age relationships between co-occurring foraminifera, alkenones, and total organic carbon in continental margin sediments. Paleoceanography 20: PA1016; doi:10.1029/2004PA001103.
Ohkouchi N, Eglinton TI, Keigwin LD, Hayes JM. 2002. Spatial and temporal offsets between proxy records in a sediment drift. Science 298:1224-7.

Ohkouchi N, Eglinton TI, Hayes JM. 2003. Radiocarbon dating of individual fatty acids as a tool for refining Antarctic margin sediment chronologies. Radiocarbon 45(1):17-24.

Ohkouchi N, Xu L, Reddy CM, Montluçon D, Eglinton TI. 2005. Radiocarbon dating of alkenones from marine sediments: I. Isolation protocol. Radiocarbon, this issue, $\mathrm{p}$ 401-12.

Pearson A, Eglinton TI. 2000. The origin of n-alkanes in Santa Monica Basin surface sediment: a model based on compound-specific $\Delta^{14} \mathrm{C}$ and $\delta^{13} \mathrm{C}$. Organic Geochemistry 31:1103-16.

Pearson A, McNichol A, Schneider RJ, von Reden KF, Zheng Y. 1998. Microscale AMS ${ }^{14} \mathrm{C}$ measurement at NOSAMS. Radiocarbon 40(1):61-75.

Pearson A, Eglinton TI, McNichol A. 2000. An organic tracer for surface ocean radiocarbon. Paleoceanography 15:541-50.

Prahl FG, Wolfe GV, Sparrow MA. 2003. Physiological impacts on alkenone paleothermometry. Paleoceanography 18: doi:10.1029/2002PA000803.

Reddy CM, Pearson A, Xu L, McNichol AP, Benner BA, Wise SA, Klouda GA, Currie LA, Eglinton TI. 2002. Radiocarbon as a tool to apportion the sources of polycyclic aromatic hydrocarbons and black carbon in environmental samples. Environmental Science and Technology 36:1774-82.

Smittenberg RH, Hopmans EC, Schouten S, Hayes JM, Eglinton TI, Sinninghe Damsté JS. 2004. Compoundspecific radiocarbon dating of the varved Holocene sedimentary record of Saanich Inlet, Canada. Paleoceanography 19: doi:10.1029/2003PA000927.

Teuten EL, Xu L, Reddy CM. 2005. Two abundant bioaccumulated halogenated compounds are natural products. Science 307:917-20.

von Reden K, McNichol A, Pearson A, Schneider RJ. 1998. ${ }^{14} \mathrm{C}$ AMS measurements of $<100 \mu \mathrm{g}$ samples with a high-current system. Radiocarbon 40(1):24753. 